\title{
Some non-intuitive properties of simple extensions of the chemostat model
}

\author{
Alain Rapaport \\ UMR 729 INRA/SupAgro MISTEA (Mathématiques, Informatique et STatistique pour \\ l'Environnement et l'Agronomie), 2 pl. Viala, 34060 Montpellier, France
}

\begin{abstract}
We show how the consideration of two compartments in the well known chemostat model could lead to non-intuitive messages in terms of performances and stability. These compartments and their interconnections represent spatial patterns and interplay with species biodiversity. The case of an inhibited resource is also considered, for which we also study the effect of a bio-augmentation.

Keywords: chemostat, compartments, interconnections, competition, growth inhibition, performances, stability, bio-augmentation.
\end{abstract}

\section{Introduction}

The chemostat model appears in the fifteens as the mathematical representation of the microbial growth in the chemostat experimental device, invented simultaneously by Monod [1] and Novick-Szilard [2]. If $s$ and $x$ denote respec-

5 tively the substrate and biomass concentrations in a culture vessel of volume $V$, their time evolution are modeled by the following system of ordinary differential equations

$$
\begin{aligned}
\dot{s} & =-\frac{1}{Y} \mu(s) x+-\frac{Q}{V}\left(S_{i n}-s\right) \\
\dot{x} & =\mu(s) x-\frac{Q}{V} x
\end{aligned}
$$

Email address: alain.rapaport@inra.fr (Alain Rapaport) 
where $Y{ }^{1}$ is the yield conversion of substrate into biomass, $\mu(\cdot)$ the specific growth rate of the micro-organisms (which is non-negative function, null only 10 at $s=0), Q$ the input flow and $S_{i n}$ the input concentration of substrate. Later, this model has been used to represent many other ecosystems in natural environments [3, 4, 5, which have in common a continuous culture of microorganisms. This model (or close versions of it) is often found in bio-mathematics, theoretical ecology or bio-processes literature (see for instance [6, 7, 8, 9]). More generally, it is a popular model of resource-consumer in living sciences (although the word "chemostat" is not always used).

The model (1) is based on several essential assumptions: 1. The microorganisms introduced in the vessel are of a single species; 2 . The substrate (of concentration $s$ ) is the single limiting resource for growth; 3 . The vessel is 20 perfectly mixed; 4 . Its volume is constant (i.e. the input and output flows are both equal to $Q$. Many extensions of this model have been studied to better suit real ecosystems, introducing considerations such as species competition, multiple limiting resources, non-perfectly mixed medium... Most of the studies in bio-mathematics and theoretical ecology have been conducted with the objective to characterize the composition and the spatial distribution of the asymptotic solutions (see for instance [10, 11, 12, 13, 14]). The performances of the related ecosystems are usually not addressed in those theoretical studies. This is different in bio-processes literature. Optimizing the performances is of primer interest, but mixed cultures are rarely considered.

The objective of the present paper is to review studies of extensions of the model (1) that have revealed "non-intuitive" messages (in the sense that the conclusions cannot be deduced straightforwardly from the equations) related to the performances. We do not pretend here to be exhaustive but we focus on situations for which a certain kind of complexity could emerge from quite 35 simple ecosystems. More precisely, we consider structures of the model (1) in a

\footnotetext{
${ }^{1}$ without any loss of generality one can assume $Y=1$ in equations 1 by simply denoting the quantity $X / Y$ by $X$
} 
few compartments that can change radically the behavior of the solutions and its performances. The consideration of spatial compartments in the chemostat model (also named gradostat which refers to the experimental device proposed by Lovitt and Wimpenny [15]) is not new [16, 17, 18, 19, 20, 21, 11, 22, 23,

40 13, 24, 25, 14, but we focus here on the output performances of the systems. From another view point, interconnected chemostats are often considered in biotechnology for optimizing the productivity [26, 27, 28, 29, 30, 31] but most of the time the configurations are in series with pure culture. In the present work, we consider more general interconnection structures and the possibilities of having several species in different niches. The paper is organized as follows. In Section 2 we analyze the effect of a spatialisation described in terms of interconnected compartments, and show how interconnection patterns could impact the performances. In Section 3 we study the interest for having a diversity in the compartments. In Section 4, we consider inhibitory resources and demonstrate the role of patterns on the ecosystem stability and its performances. Finally, in Section 5 , the bio-augmentation is analyzed in terms of another mean that could impact the stability of the ecosystem.

\section{Spatial patterns in the chemostat}

In this Section, we assume that the growth function $\mu(\cdot)$ is monotonically increasing. A usual function is given by the Monod's expression (see Figure 11) in the Appendix):

$$
\mu(s)=\mu_{\max } \frac{s}{K_{s}+s} .
$$

For convenience we denote the dilution rate $D=\frac{Q}{V}$, and define, as it is often made in the literature, the break-even concentration associated to the growth function as

$$
\lambda(D)=\mid \begin{array}{ll}
s \text { s.t. } \mu(s)=D & \text { when } \max _{s} \mu(s)>D, \\
+\infty & \text { otherwise. }
\end{array}
$$

60 Let us first recall the classical results about the asymptotic behavior of the solutions of the model (1) (see for instance [12]). The equilibria are the wash-out 
$E_{0}=\left(S_{i n}, 0\right)$ and a positive steady state $E_{1}=\left(\lambda(D), S_{i n}-\lambda(D)\right)$ which exists when $\lambda(D)<S_{i n}$. Under this last condition, $E_{0}$ is unstable and any solution with $x(0)>0$ converges asymptotically to $E_{1}$. On the contrary, when $\lambda(D) \geq$ $S_{i n}$, any solution converges asymptotically to $E_{0}$. Therefore, a property of the model (1) is that for a given dilution rate $D$, the output substrate concentration at steady state is equal to $\lambda(D)$ independently of the input concentration $S_{i n}$, provided $S_{i n}$ to be larger to $\lambda(D)$. It is well known that this property is no longer satisfied when there is spatial heterogeneity (see for instance [15, 27] where expressions of the output concentrations at steady state depend on the input concentration). Performances of an ecosystem can be measured by different indexes. We consider here an index that measures the ability of an ecosystem to convert a resource. More precisely, for a given value $S_{i n}$ of the resource density at the inlet, we define its output density at steady state, denoted $s_{\text {out }}^{\star}$, as the performance index. To grasp the effect of spatial structures on this performance index, we consider three patterns depicted on Figure 1 for a given total volume $V$ and input flow rate $Q$. We compare the smallest value $s_{\text {out }}^{\star}$

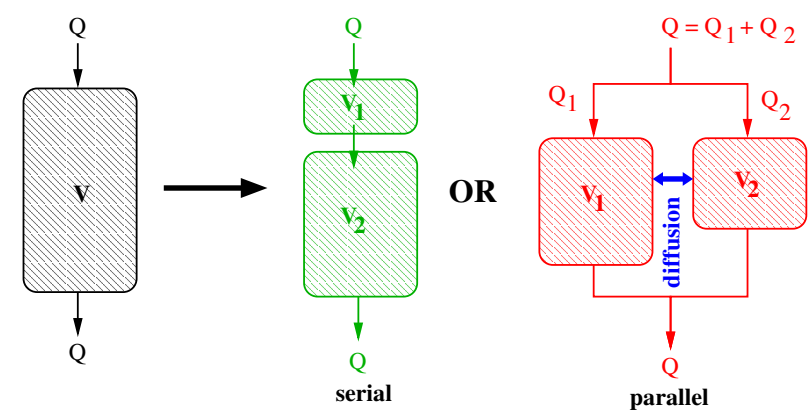

Figure 1: Consideration of three spatial patterns with same total volume $V$.

for each configuration. For the single tank configuration, $s_{\text {out }}^{\star}$ is simply given by $\lambda(Q / V)$. For the serial configuration, $s_{\text {out }}^{\star}$ is function of the volume ratio so $r=V_{1} / V$. For the parallel configuration, $s_{\text {out }}^{\star}$ is function of $r$, the ratio of flow distribution $\alpha=Q_{1} / Q$ and the diffusion parameter $d$ between the two volumes. The systems of equations (13) and (14) for the serial in parallel configurations are given in Appendix. One has the following result. 
Proposition 1. For a given input flow rate $Q$ and volume $V$, there exists a threshold $\bar{S}_{i n}>0$ such that the smallest output concentration $s_{\text {out }}^{\star}$ is reached for a serial configuration when $S_{i n}>\bar{S}_{i n}$, and for a parallel configuration when $S_{\text {in }}<\bar{S}_{\text {in. }}$. Moreover, in this last case, the map $d \mapsto s_{\text {out }}^{\star}(d)$ admits an unique minimum for a $d^{\star}<+\infty$ Furthermore, there exists another threshold $\underline{S}_{i n}<\bar{S}_{i n}$ such that $d^{\star}=0$ for $S_{i n}<\underline{S}_{\text {in }}$ and $d^{\star}>0$ for $S_{\text {in }} \in\left(\underline{S}_{i n}, \bar{S}_{i n}\right)$.

90 These results have been proved in [32] for linear growth functions and later extended to Monod functions in 33. We illustrate these results for a linear growth function with total volume $V$ and input flow rate $Q$ such that $\lambda(Q / V)=$ 1. For the single tank configuration, the output concentration at steady state is thus equal to 1 . The threshold $\bar{S}_{\text {in }}$ can be computed to be equal to 2 . On

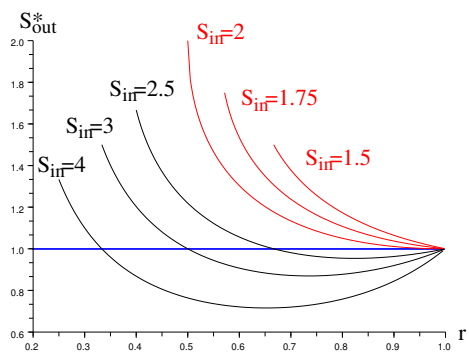

serial

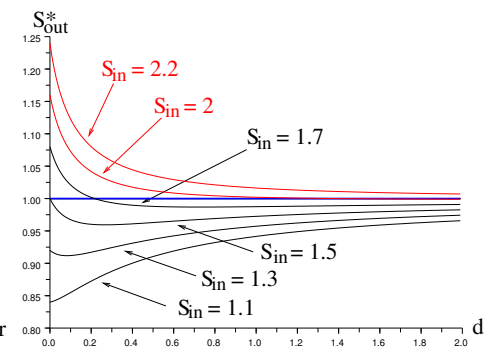

parallel

Figure 2: Output performances of the serial and parallel configurations. various values of $S_{i n}$ as function of the parameters of the serial or parallel configurations. On can first see that for any value of $S_{i n}$, there always exists a serial or parallel configuration such that $s_{\text {out }}^{\star}<1$ (that is consequently better than having a single tank). When $S_{i n}$ is above the threshold $\bar{S}_{i n}=2$, the that the serial configuration has $s_{\text {out }}^{\star}$ under 1 . For $S_{\text {in }}$ below the threshold, conclusions are reversed: the serial configuration has $s_{\text {out }}^{\star}$ always larger than 1 , while there exist parameter values of $d$ such that the parallel configuration 
has $s_{\text {out }}^{\star}$ lower than 1. Moreover, one can see that for values of $S_{\text {in }}$ under the threshold but no too low, the smallest value of $s_{\text {out }}^{\star}$ is obtained for a positive value of the diffusion parameter $d$.

\section{Biodiversity and spatial patterns}

Now we consider the case of two species (or two consumers) of concentrations $x_{1}, x_{2}$ in competition for the single limiting resource in the chemostat. A straightforward extension of the mono-specific model (1) is given by the system (where the yield parameter $Y$ has been kept equal to 1)

$$
\begin{aligned}
\dot{s} & =-\mu_{a}(s) x_{a}-\mu_{b}(s) x_{b}-D\left(S_{i n}-s\right) \\
\dot{x}_{a} & =\mu_{a}(s) x_{a}-D x_{a} \\
\dot{x}_{b} & =\mu_{b}(s) x_{b}-D x_{b}
\end{aligned}
$$

where $D=Q / V$ is the dilution rate, as before. On can see, from the two last equations, that a coexistence of both species at steady state would imply the existence of a (positive) steady state value $s^{\star}$ such that $\mu_{a}\left(s^{\star}\right)=\mu_{b}\left(s^{\star}\right)=D$. This is a very restrictive condition that cannot be met generically. If the graphs of the functions $\mu_{a}(\cdot), \mu_{b}(\cdot)$ do not cross away from 0 , this condition cannot be fulfilled. Otherwise, the condition can be fulfilled at the graphs intersections, which are generically isolated points. Therefore, such a condition can be satisfied at most at some isolated values of $D$, which are hardly possible to observe in practice. This is the core of the well known Competitive Exclusion Principle (CEP) that states moreover that the species $i \in\{a, b\}$ with the smallest breakeven concentration $\lambda_{i}(D)$ wins the competition (when $\lambda_{i}(D)<S_{\text {in }}$, otherwise both species are washed-out). This is a particular case of a more general result that was proved for an arbitrary number of species in [10] (for Monod functions) and in [36] (for any monotonic growth functions). Note that the winning species could depend on the value of the dilution rate $D$ when the graphs of the two growth functions $\mu_{a}(\cdot), \mu_{b}(\cdot)$ intersect away from 0 , as depicted on Figure 3(a) for two Monod functions, that is when the following assumption is fulfilled. 
Hypothesis 1. (Crossing assumption) There exists $\bar{D}>0$ such that

$$
D>0, D \neq \bar{D} \Rightarrow\left(\lambda_{a}(D)-\lambda_{b}(D)\right)(D-\bar{D})>0
$$

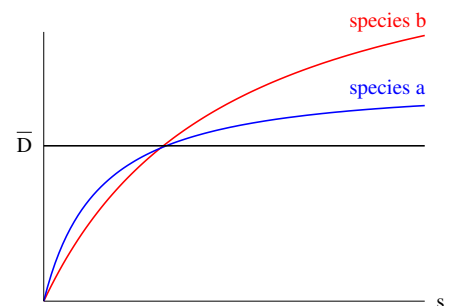

(a) When $D<\bar{D}$, the species $a$ wins, while species $b$ wins when $D>\bar{D}$

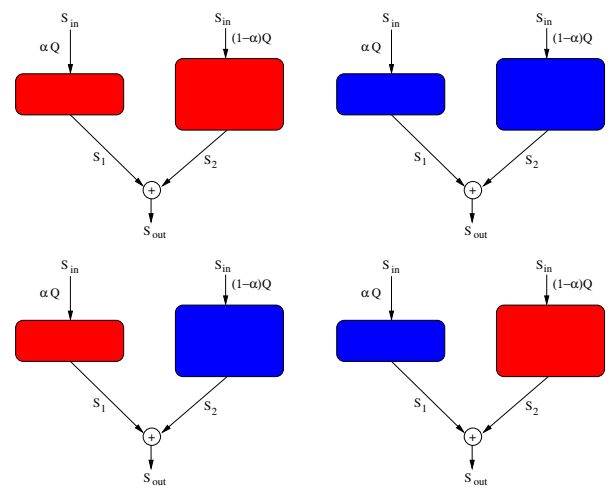

(b) Four possibilities of occupancy by a single species in each tank, according to the CEP

Figure 3: The case of two species under the crossing assumption.

In 34 the question of an optimal design of two tanks in series has been addressed with two species in competition under the crossing assumption (the equations (15) of the system are given in the Appendix). In the first tank, the equations are exactly (3) with $D$ replaced by $Q_{1} / V_{1}$. Therefore, the CEP holds: either species $a$ or $b$ wins (on the condition that the volume $V_{1}$ is such that $\left.\min _{i=a, b} \lambda_{i}\left(Q_{1} / V_{1}\right)<S_{i n}\right)$. In the second tank, the CEP cannot be applied due to the inlet of the winning species from the first tank. In [34, it is shown that a coexistence of two species is possible in the second tank, but the optimal distribution of the volumes cannot sustain both species. Moreover the optimal solution for the bio-conversion performance is robust with respect to invasion, in the sense than a less efficient species cannot invade the ecosystem, while a better species replaces the existing one. For the parallel configuration, one has the exclusion of one (or both) species in each tank when the tanks are isolated (i.e. with no diffusion). The four options are depicting on Figure 3(b) depending on which species has been chosen in each tank. The equations (16) of the dynamics are given in the Appendix. 
For convenience, we denote the modified break-even concentration

$$
\bar{\lambda}_{i}(D)=\min \left(\lambda_{i}(D), S_{i n}\right), \quad i=a, b .
$$

Then, the output concentration at steady state when species $i$ and $j$ are present in the first and second tanks, respectively, is given by

$$
F_{i, j}(r, \alpha)=\alpha \bar{\lambda}_{i}\left(\frac{\alpha}{r} D\right)+(1-\alpha) \bar{\lambda}_{j}\left(\frac{1-\alpha}{1-r} D\right)
$$

where $r$ denotes the ratio $V_{1} / V$, as before. We look for situations for which having different species in each compartment is beneficial for the performance, according to the following definition.

Definition 1. (Over-yielding). A configuration $(r, \alpha)$ presents over-yielding when there exits $i \neq j$ such that

$$
F_{i, j}(r, \alpha)<\min \left(F_{a, a}(r, \alpha), F_{b, b}(r, \alpha)\right) .
$$

Consider $Q$ and $V$ such that $Q / V=\bar{D}$, and a configuration $(r, \alpha)$ with $\alpha / r<1$. One has necessarily $(1-\alpha) /(1-r)>1$. Denote then $\bar{D}_{1}=(\alpha / r) \bar{D}$ and $\bar{D}_{2}=(1-\alpha) /(1-r) \bar{D}$ the dilution rates for the two compartments. If $\bar{D}_{2}<$ $\min \left(\mu_{1}\left(S_{i n}\right), \mu_{2}\left(S_{i n}\right)\right.$, one has $\bar{\lambda}_{a}\left(\bar{D}_{1}\right)<\bar{\lambda}_{a}\left(\bar{D}_{1}\right)$ and $\bar{\lambda}_{b}\left(\bar{D}_{2}\right)<\bar{\lambda}_{a}\left(\bar{D}_{2}\right)$. Then, one obtains

$$
\begin{aligned}
F_{a, b}(r, \alpha) & =\frac{\alpha}{r} \bar{\lambda}_{a}\left(\bar{D}_{1}\right)+\frac{1-\alpha}{1-r} \bar{\lambda}_{b}\left(\bar{D}_{2}\right) \\
& <\frac{\alpha}{r} \bar{\lambda}_{b}\left(\bar{D}_{1}\right)+\frac{1-\alpha}{1-r} \bar{\lambda}_{b}\left(\bar{D}_{2}\right)=F_{b, b}(r, \alpha)
\end{aligned}
$$

and similarly $F_{a, b}(r, \alpha)<F_{a, a}(r, \alpha)$. So an over-yielding occurs for the particular value of $D=\bar{D}$. One can show that this also occurs for any $D$ not too far from $\bar{D}$, as stated in the following result.

Proposition 2. From Assumption 1, over-yielding occurs for $(r, \alpha)$ such that

$$
\frac{\alpha}{r} D<\bar{D}<\frac{1-\alpha}{1-r} D<\min \left(\mu_{a}\left(S_{i n}\right), \mu_{b}\left(S_{i n}\right)\right) .
$$

We illustrate this result considering two Monod functions with an intersection at a common (positive) value $\bar{D}=\mu_{a}(\bar{s})=\mu_{b}(\bar{s})$ for some $\bar{s}$. For a value $D$ close 
to $\bar{D}$, we compare the options of having the same species ( $a$ or $b$ ) in both tanks or having having different species in the two tanks: Figure 4 shows the graphs of $F_{a, b}(\cdot), F_{a, a}(\cdot), F_{b, b}(\cdot)$ as functions of $\alpha$ for a fixed value of $r$. One can see that the values of $F_{a, b}(\cdot)$ are significantly below the minima of $F_{a, a}(\cdot)$ and $F_{b, b}(\cdot)$ (more precisely a gain of $12 \%$ is obtained on this example). This demonstrates the benefit of having different species in the two tanks, which play then the role of two niches. One can also remark that the functions $F_{i, j}$ depicted on Figure

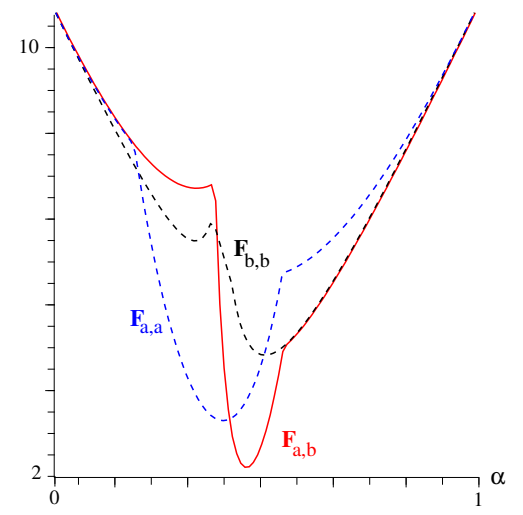

Figure 4: $F_{i, j}$ as function of $\alpha$ for $r=0.4$, with $\mu_{a}(s)=2 s /(3+s), \mu_{b}(s)=s /(0.3+s)$, $D=0.9$ and $S_{\text {in }}=10$.

4 are non-convex, although the functions $\lambda_{i}$ are convex (when the functions $\mu_{i}$ are concave, which is the case for Monod functions). This comes from the fact that one has to consider the saturated break-even functions $\bar{\lambda}_{i}$ in the definition of the performance index $F_{i, j}$.

\section{Inhibitory resources in the chemostat}

In this Section we consider non-monotonic growth functions $\mu(\cdot)$, that are increasing for values of $s$ up a threshold $s_{m}$ and decreasing for values of $s$ larger of $s_{m}$. A typical instance is given by the Haldane function (see Figure 11) in the Appendix).

$$
\mu(s)=\bar{\mu} \frac{s}{K_{s}+s+s^{2} / K_{i}} .
$$


Andrews has shown that such functions well represent a growth inhibited by large densities of the resource [35]. For such functions, the concept of breakeven concentration recalled in Section 2 has to be revisited. Following [36, we consider the interval

$$
I(D)=\{s>0 \mid \mu(s)>D\}
$$

185

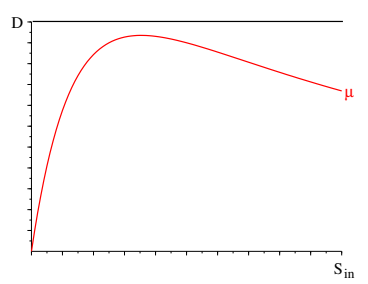

(a) no positive equilibrium

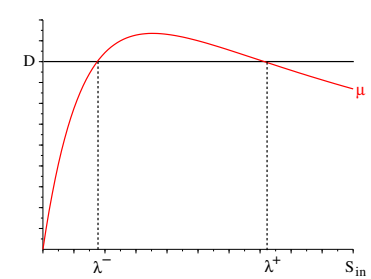

(b) 2 positive equilibria

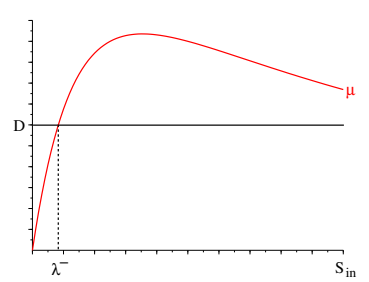

(c) 1 positive equilibrium

Figure 5: Consideration of the Haldane function in the chemostat model.

The second case can faces irreversibility when a sudden drop of the biomass occurs, due for instance to temporary toxicity or harvesting. It can lead the state to the attraction basin of the wash-out equilibrium. Contrary to the monotonic case, the biomass cannot recover from such event and finally dies. 
One may then wonder what role a spatialisation could play concerning this instability.

Given $Q$ and $V$, we consider rich environment, that is for $S_{\text {in }}>\lambda^{+}(D)$. If one considers a serial configuration, the wash-out equilibrium stays attraccompartments (without diffusion) that allows to preserve robustly biomass in both compartment, that we call buffered connection as drawn on Figure6. The

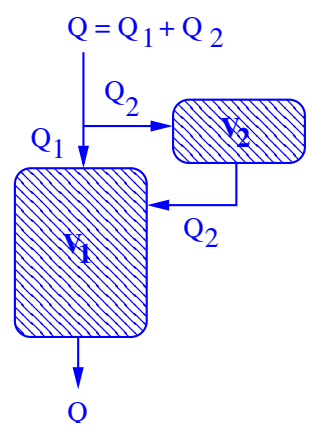

Figure 6: The buffered chemostat.

equations (17) of the dynamics of this configuration are given in the Appendix. It is convenient to parameterize this configuration with the pair $(r, \alpha)$ defined 215 as $r=V_{1} / V$ and $\alpha=\left(Q_{2} / V_{2}\right) / D$. We note that the second tank has a single input and therefore behaves as a single chemostat. Let us fix $\alpha<1$ such that $Q_{2} / V_{2}=\alpha D<\mu\left(S_{i n}\right)$ guarantees to have a single positive state $\left(s_{2}^{\star}, x_{2}^{\star}\right)$ with $s_{2}^{\star}=\lambda^{-}\left(Q_{2} / V_{2}\right)$ and $x_{2}^{\star}=S_{i n}-s_{2}^{\star}$, as in case 3 above. Note that at this stage we have not yet chosen $Q_{2}$ and $V_{2}$ but simply the ratio $Q_{2} / V_{2}$. Then, 220

For the parallel configuration (with no diffusion) a dilution rate $Q_{1} / V_{1}$ small enough makes the wash-out equilibrium repulsive in the first compartment, but one can easily check that this implies to have the dilution rate $Q_{2} / V_{2}$ larger than $D$ and thus the wash-out equilibrium is attractive in the second compartment. However, we show that there exists another configuration with two 
tank with $s_{1}^{\star}$ solution of the equation

$$
\mu\left(s_{1}^{\star}\right)=\frac{D}{r}-\alpha D\left(1-\frac{1}{r}\right) \frac{S_{i n}-s_{2}^{\star}}{S_{i n}-s_{1}^{\star}}:=\phi_{r}\left(s_{1}^{\star}\right)
$$

along with $x_{1}^{\star}=S_{i n}-s_{1}^{\star}$. The solutions of this equation can be graphically interpreted as the intersection of the graphs of the functions $\mu(\cdot)$ and $\phi_{r}(\cdot)$. It has been shown in 37] that the graphs of the family of functions $\phi_{r}(\cdot)$ for ${ }_{225} r \in(0,1)$ have the point $\left(\bar{s}_{1}, D\right)$ in common, where $\bar{s}_{1}=\alpha s_{2}^{\star}+(1-\alpha) S_{i n}$. Figure 7 depicts the family $\phi_{r}(\cdot)$ and the intersections with $\mu(\cdot)$, depending on the position of $\bar{s}_{1}$ with respect to $\lambda^{+}(D)$. One can see that among this family
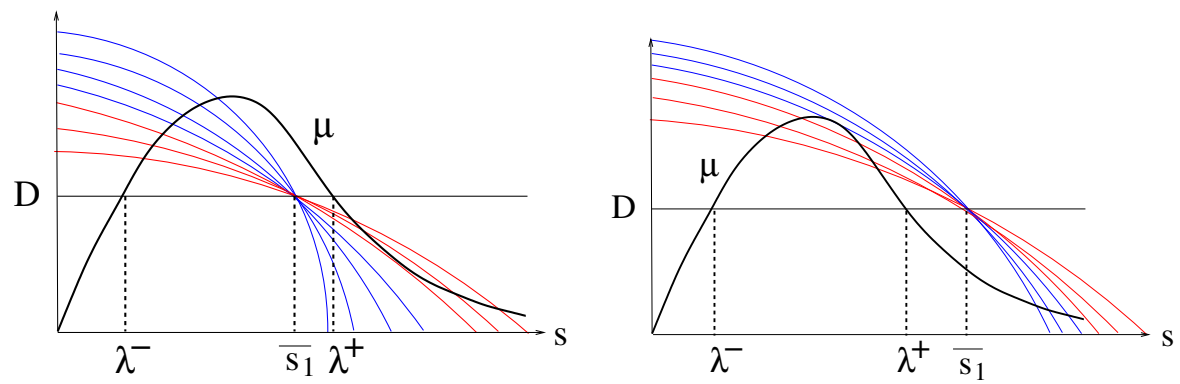

Figure 7: Family of functions $\phi_{r}(\cdot)$ (in blue single intersection with the function $\mu$ ).

there always exist functions $\phi_{r}(\cdot)$ whose graph possesses a unique intersection with the graph of $\mu(\cdot)$. For such cases, there is a unique positive equilibrium in the first tank as well. In [37, the following result is given.

Proposition 3. Assume $S_{\text {in }}>\lambda^{+}(D)$. For any $\alpha \in(0,1)$ such that $\alpha D<$ $\mu\left(S_{i n}\right)$, there exists $r \in(0,1)$ such that buffered chemostat configuration with $(r, \alpha)$ has an unique positive equilibrium. Moreover, for any initial condition with $x_{2}(0)>0$, the solution converges asymptotically to this equilibrium. One can also see on Figure 7 that the price to pay for stability with a buffered interconnection is that the output concentration of the resource $s_{\text {out }}^{\star}$ as steady state (which is equal to $s_{1}^{\star}$ ) is larger than $\lambda^{-}(D)$, which is the output concentration of the single chemostat of volume $V$ at the positive equilibrium. In [37, the problem of minimizing $s_{\text {out }}^{\star}$ among all the buffered configurations that ensure global stability is also studied. The following result is provided in [37. 
Proposition 4. Assume $S_{\text {in }}>\lambda^{+}(D)$. The configuration $\left(r^{\star}, \alpha^{\star}\right)$ minimizing the output substrate concentration $s_{\text {out }}^{\star}(r, \alpha)$ at steady state among all the globally stable configurations fulfills the following properties.

1. One has $\alpha^{\star}=\mu\left(s^{\star}\right)$ where $s^{\star}$ maximizes the function

$$
\psi(s)=\mu(s)\left(S_{i n}-s\right)
$$
on the interval $\left[0, \lambda^{-}\left(\mu\left(S_{\text {in }}\right)\right]\right.$.

2. There exists a threshold $\bar{S}_{\text {in }}>0$ defined as

$$
\max _{s \in\left[0, \lambda^{-}\left(\mu\left(\bar{S}_{i n}\right)\right]\right.} \psi(s)=\bar{S}_{i n}-\lambda^{+}(D)
$$

such that $r^{\star} \in(0,1)$ when $S_{\text {in }}$ is under this threshold and $r^{\star}=1$ when $S_{i n}$ is above.

Indeed, the threshold $\bar{S}_{i n}$ corresponds to the limiting case for which it is not possible to have an unique $s_{1}^{\star}$ solution of (11) with $s_{1^{\star}} \leq \bar{s}_{1}$ for $\alpha=\alpha^{\star}$. Then, a configuration with a single tank $(r=1)$ and a by-pass (i.e. $Q_{1}$ such that $Q_{1} / V<\mu\left(S_{i n}\right)$ passes through the tank while $Q_{2}$ by-passes it) is the most efficient way to ensure a global stability of the system. This amounts to say that for very rich environment a global stability is quite penalizing in terms of performances (see Figure 8).

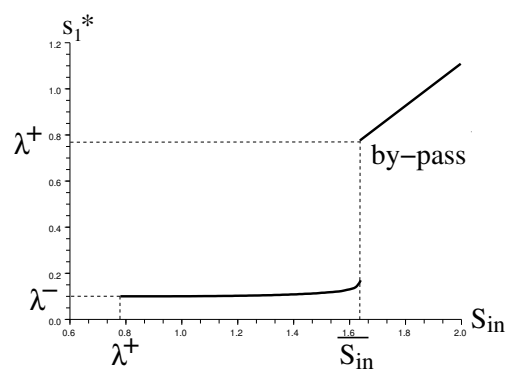

Figure 8: Best output concentration that ensures a global stability as function of the input concentration. 


\section{Bio-augmentation and stability}

In this Section, we consider a species $a$ for which the resource is inhibitory, as in Section 4, with input parameters $\left(D, S_{i n}\right)$ such that $S_{i n}>\lambda_{a}^{+}(D)$. For such case, we study the effect of adding a species $b$ on the stability of the ecosystem. One cannot have (generically) both species at steady state, as explained in Section 3 but differently to the monotonic case, the winning species could depend on the initial condition. Denote the possible equilibria $E_{0}=\left(S_{i n}, 0,0\right), E_{a}^{\mp}=$ $\left(\lambda_{a}^{\mp}(D), S_{i n}-\lambda_{a}^{\mp}(D), 0\right), E_{b}^{\mp}=\left(\lambda_{b}^{\mp}(D), 0, S_{i n}-\lambda_{b}^{\mp}(D)\right)$. One has the following result.

Proposition 5. Assume $S_{\text {in }}>\lambda_{a}^{+}(D)$ and $\lambda_{b}^{-}(D) \neq \lambda_{a}^{-}(D)$. Let $i^{\star} \in\{a, b\}$ be such that $\lambda_{i}^{-}(D)=\min \left(\lambda_{a}^{-}(D), \lambda_{b}^{-}(D)\right)$, and consider the set

$$
I=\left(I_{a}(D) \cup I_{b}(D)\right) \cap\left[0, S_{i n}\right] .
$$

1. If $I$ is an interval with $S_{i n} \in I, E_{i^{\star}}^{-}$is the unique stable equilibrium that attracts any solution with $x_{a}(0)>0$ and $x_{b}(0)>0$.

2. If $I$ is an interval with $S_{\text {in }} \notin I$, there is a bi-stability between $E_{0}$ and $E_{i^{\star}}^{-}$.

3. If $I$ is not connected with $S_{\text {in }} \in I$, there is a bi-stability between $E_{a}^{-}$and $E_{b}^{-}$.

4. If I is not connected with $S_{\text {in }} \notin I$, there is a tri-stability between $E_{0}, E_{a}^{-}$ and $E_{b}^{-}$.

This result has been proved in [36] and later extended in [38, 39, 40, for different removal rates.

Let the species $a$ be alone with a initial condition in the attraction basin of the wash-out equilibrium: species $a$ goes to the wash-out (see Figure 10(a)). Consider a bio-augmentation at initial time with a species $b$ whose growth function is monotonic but less efficient at steady state (i.e. such that $\lambda_{b}(D)>$ $\left.{ }_{280} \lambda_{a}^{-}(D)\right)$. Figure 9 shows two possible candidates for the species $b$ to compete with $a$. Species $b$ (in green) is more efficient than species $c$ (in blue).

The simulations presented on Figure 10 have the same initial conditions $s(0), x_{a}(0)$ but with an additional small quantity $x_{b}(0)>0$ on Figures $10(\mathrm{~b})$ 

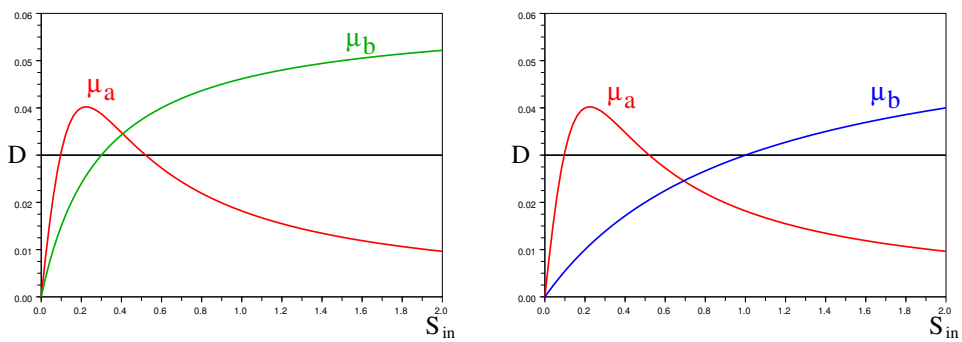

Figure 9: Consideration of bio-augmentation with the green or the blue species.

10(c) On Figure 10(b) species $b$ has been added. We are in the case 1 . of

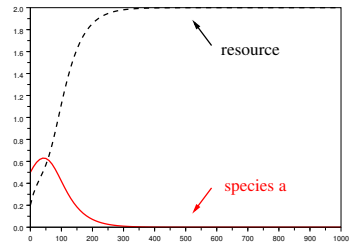

(a) species a alone

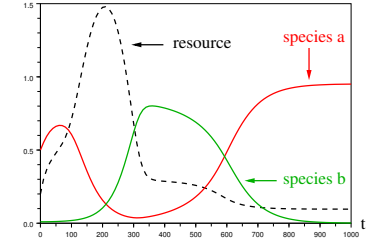

(b) bio-augmentation

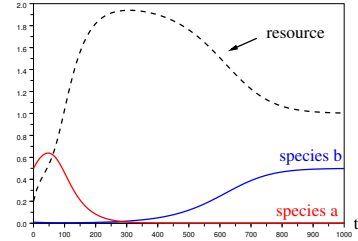

(c) bio-augmentation

Figure 10: Effect of a bio-augmentation.

Proposition 5. Any small initial quantity $s_{b}(0)$ is enough to allow species $a$ to survive and then win the competition. Therefore, the presence of species $b$ is necessary to avoid the wash-out of species $a$, but it is asymptotically eliminated from the ecosystem. A bio-control strategy could then consist in a regular input of species $b$ for guaranteeing a recovery of the performances of the ecosystem 290 in case of a sudden drop of species $a$. On Figure 10(c) species $c$ is added instead of species $b$. We are in the case 3. of Proposition 5 species $c$ could win the competition, depending on the initial condition. The performances of the ecosystem is irreversibly impacted, compared to the stable equilibrium with species $a$. At the first look, it could appear paradoxical that species $c$ can settle in the chemostat, while the more efficient species $b$ cannot. An interpretation is that species $c$ being less efficient then species $b$, is not fast enough to consume the resource for avoiding the species $a$ to be washed-out. 


\section{Concluding messages}

The present study highlights several messages in terms of input-output per-

300

It can be summarized as follows.

- There always exist spatial distributions which improve the substrate conversion compared to a single perfectly mixed volume. For rich environments (i.e. for large value of $S_{i n}$ ), a serial distribution can be the most

305 well-known chemostat model could reveal properties, that we consider to be non-intuitive, in terms of performances and stability. This strengthens the contribution of mathematical analysis for a deeper understanding, that could be applied on similar or closed models, such as the ones used for instance for soil 325

efficient, while for a poor environment a parallel distribution can be the best, and a moderate diffusion could even improve it.

- While spatial patterns could allow to have different species present in the ecosystem at steady state, there is no interest of having more than one species in serial configurations in view of performances. Situation is much different for parallel configurations. Under the crossing assumption, one can have two niches in parallel with a different species in each niche that together have a better utilization of the resource than having the same species in each niche.

- In presence of inhibition, particular spatial patterns such as the buffered interconnection could bring global stability instead of bi-stability, but at the price of a trade-off with its performances.

- Bio-augmentation could be another mean to obtain a global stability in presence of inhibition, allowing to recover the initial performances in case of a sudden drop of the most efficient species.

Finally, we have shown that the consideration of simple extensions of the ecosystems or epidemiology. 


\section{Acknowledgment}

The author thanks the organizers of the Conference on Models in Population Dynamics and Ecology (MPDE'16), Marseille, France, Sep. 2016, for the opportunity to give a plenary talk.

\section{References}

[1] J. Monod, La technique de culture continue : Théorie et applications, Ann. Inst. Pasteur, Lille 79 (1950) 390-410.

[2] A. Novick, L. Szilard, Experiments with the chemostat on spontaneous mutations of bacteria, Proceedings of the National Academy of Sciences 36 (12) (1950) 708-719.

[3] A. Hasler, W. Johnson, The in situ chemostat - a self-contained continuous culturing and water sampling system, Limnol. Oceanogr. 79 (1954) 326331.

[4] H. Veldcamp, Ecological studies with the chemostat, Advances in Microbial Ecology 1 (1977) 59-95.

[5] Y. Higashi, N. Ytow, H. Saida, H. Seki, In situ gradostat for the study of natural phytoplankton community with an experimental nutrient gradient, Environmental Pollution 99 (1998) 395-404.

[6] S. Pirt, Principles of microbe and cell cultivation, Blackwell Scientific Publications, 1975.

[7] N. Panikov, Microbial Growth Kinetics, Springer Netherlands, 1995.

[8] A. Koch, J. Robinson, G. Milliken, Mathematical Modeling in Microbial Ecology, Chapman \& Hall, 1998.

[9] G. Bastin, D. Dochain, On-Line Estimation and Control of Bioreactors, Elsevier Science Publishers, Amsterdam, 1990. 
[10] S. Hsu, S. Hubbell, P. Waltman, A mathematical theory for single-nutrient competition in continuous cultures of microorganisms, SIAM Journal on Applied Mathematics 32 (1977) 366-383.

[11] J. Hofbauer, W. So, Competition in the gradostat: the global stability problem, Nonlinear Analysis: Theory, Methods \& Applications 8 (22) (1994) $1017-1031$.

[12] H. L. Smith, P. Waltman, The theory of the chemostat: dynamics of microbial competition, Cambridge University Press, 1995.

[13] P. Amarasekare, R. Nisbet, Spatial heterogeneity, source sink dynamics, and the local coexistence of competing species, The American Naturalist 6 (158) (2001) 572-584.

[14] D. Gravel, F. Guichard, M. Loreau, N. Mouquet, Source and sink dynamics in metaecosystems, Ecology 91 (2010) 2172-2184.

[15] R. Lovitt, J. Wimpenny, The gradostat: a bidirectional compound chemostat and its application in microbiological research, J. Gen. Microbiol. 127 (1981) 261-268.

[16] G. Stephanopoulos, A. Fredrickson, Competition in chemostat-type equations with two habitats, Biotechnology and Bioengineering 21 (2006) 14911498.

370 [17] B. Tang, Mathematical investigations of growth of microorganisms in the gradostat, J. Math. Biol. 23 (1986) 319-339.

[18] W. Jaeger, J.-H. So, B. Tang, P. Waltman, Competition in the gradostat, J. Math. Biol. 25 (1987) 23-42.

[19] H. Smith, B. Tang, Competition in the gradostat: the role of the communication rate, J. Math. Biol. 27 (2) (1989) 139-165.

[20] H. Smith, B. Tang, P. Waltman, Competition in an n-vessel gradostat, SIAM J. Appl. Math. 51 (1991) 1451-1471. 
[21] H. Smith, P. Waltman, The gradostat: a model of competition along a nutrient gradient, J. Microb. Ecol. 22 (1991) 207-226.

380

[30] A. Dramé, J. Harmand, A. Rapaport, C. Lobry, Multiple steady state profiles in interconnected biological systems, Mathematical and Computer Modelling of Dynamical Systems 12 (2006) 379-393. 
[31] M. Nelson, H. Sidhu, Evaluating the performance of a cascade of two bioreactors, Chemical Engineering Science 61 (2006) 3159-3166.

[32] I. Haidar, A. Rapaport, F. Grard, Effects of spatial structure and diffusion on the performances of the chemostat, Mathematical Biosciences and Engineering 8 (4) (2011) 953-971.

[33] I. Haidar, Mathematical modeling and analysis of soil microbial dynamics, Ph.D. thesis, University Montpellier, France (2011).

[34] A. Rapaport, J. Harmand, F. Mazenc, Coexistence in the design of a series of two chemostats, Nonlinear Analysis: Real World Applications 9 (2008) $1052-1067$.

[35] J. Andrews, A mathematical model for the continuous culture of microorganisms utilizing inhibitory substrates, Biotechnology \& Bioengineering 10 (6) (1968) 707-723.

[36] G. Butler, , G. Wolkowicz, A mathematical model of the chemostat with a general class of functions describing nutrient uptake, SIAM Journal on Applied Mathematics 45 (1985) 137-151.

[37] A. Rapaport, I. Haidar, J. Harmand, Global dynamics of the buffered chemostat for a general class of growth functions, J. Mathematical Biology 71 (1) (2015) 69-98.

[38] G. Wolkowicz, Z. Lu, Global dynamics of a mathematical model of competition in the chemostat: General response functions and differential death rates, SIAM Journal on Applied Mathematics 52 (1992) 222-233.

[39] B. Li, Global asymptotic behavior of the chemostat: General response functions and differential removal rates, SIAM Journal on Applied Mathematics 59 (1998) 411-422.

[40] A. Rapaport, J. Harmand, Biological control of the chemostat with nonmonotonic response and different removal rates, Mathematical Biosciences \& Engineering 5 (3) (2008) 539-547. 


\section{Appendix}

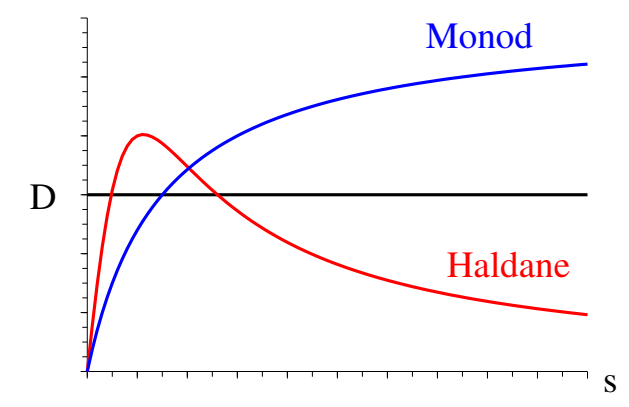

Figure 11: Graphs of Monod and Haldane functions.

The system of equations for the serial configuration is:

$$
\begin{aligned}
& \dot{s}_{1}=-\mu\left(s_{1}\right) x_{1}+\frac{Q_{1}}{V_{1}}\left(S_{i n}-s_{1}\right) \\
& \dot{x}_{1}=\mu\left(s_{1}\right) x_{1}-\frac{Q_{1}}{V_{1}} x_{1} \\
& \dot{s}_{2}=-\mu\left(s_{2}\right) x_{2}+\frac{Q_{2}}{V_{2}}\left(s_{1}-s_{2}\right) \\
& \dot{x}_{2}=\mu\left(s_{2}\right) x_{2}+\frac{Q_{2}}{V_{2}}\left(x_{1}-x_{2}\right)
\end{aligned}
$$

with $s_{\text {out }}=s_{2}$.

The system of equations for the parallel configuration is:

$$
\begin{aligned}
& \dot{s}_{1}=-\mu\left(s_{1}\right) x_{1}+\frac{Q_{1}}{V_{1}}\left(S_{i n}-s_{1}\right)+\frac{d}{V_{1}}\left(s_{2}-s_{1}\right) \\
& \dot{x}_{1}=\mu\left(s_{1}\right) x_{1}-\frac{Q_{1}}{V_{1}} x_{1}+\frac{d}{V_{1}}\left(x_{2}-x_{1}\right) \\
& \dot{s}_{2}=-\mu\left(s_{2}\right) x_{2}+\frac{Q_{2}}{V_{2}}\left(S_{i n}-s_{2}\right)+\frac{d}{V_{1}}\left(s_{1}-s_{2}\right) \\
& \dot{x}_{2}=\mu\left(s_{2}\right) x_{2}-\frac{Q_{2}}{V_{2}} x_{2}+\frac{d}{V_{1}}\left(x_{1}-x_{2}\right)
\end{aligned}
$$

with $s_{\text {out }}=\frac{Q_{1} s_{1}+Q_{2} s_{2}}{Q_{1}+Q_{2}}$. 
The system of equations for the serial configuration with two species is:

$$
\begin{aligned}
& \dot{s}_{1}=-\mu_{a}\left(s_{1}\right) x_{a, 1}-\mu_{b}\left(s_{1}\right) x_{b, 1}+\frac{Q_{1}}{V_{1}}\left(S_{i n}-s_{1}\right) \\
& \dot{x}_{a, 1}=\mu_{a}\left(s_{1}\right) x_{a, 1}-\frac{Q_{1}}{V_{1}} x_{a, 1} \\
& \dot{x}_{b, 1}=\mu_{b}\left(s_{1}\right) x_{b, 1}-\frac{Q_{1}}{V_{1}} x_{b, 1} \\
& \dot{s}_{2}=-\mu_{a}\left(s_{2}\right) x_{a, 2}-\mu_{b}\left(s_{2}\right) x_{b, 2}+\frac{Q_{2}}{V_{2}}\left(s_{1}-s_{2}\right) \\
& \dot{x}_{a, 2}=\mu_{a}\left(s_{2}\right) x_{a, 2}+\frac{Q_{2}}{V_{2}}\left(x_{a, 1}-x_{a, 2}\right) \\
& \dot{x}_{b, 2}=\mu_{b}\left(s_{2}\right) x_{b, 2}+\frac{Q_{2}}{V_{2}}\left(x_{b, 1}-x_{b, 2}\right)
\end{aligned}
$$

$440 \quad$ with $s_{\text {out }}=s_{2}$.

The system of equations for the parallel configuration (with no diffusion) is:

$$
\begin{aligned}
& \dot{s}_{1}=-\mu_{a}\left(s_{1}\right) x_{a, 1}-\mu_{b}\left(s_{1}\right) x_{b, 1}+\frac{Q_{1}}{V_{1}}\left(S_{i n}-s_{1}\right) \\
& \dot{x}_{a, 1}=\mu_{a}\left(s_{1}\right) x_{a, 1}-\frac{Q_{1}}{V_{1}} x_{a, 1} \\
& \dot{x}_{b, 1}=\mu_{b}\left(s_{1}\right) x_{b, 1}-\frac{Q_{1}}{V_{1}} x_{b, 1} \\
& \dot{s}_{2}=-\mu_{a}\left(s_{2}\right) x_{a, 2}-\mu_{b}\left(s_{2}\right) x_{b, 2}+\frac{Q_{2}}{V_{2}}\left(S_{i n}-s_{2}\right) \\
& \dot{x}_{a, 2}=\mu_{a}\left(s_{2}\right) x_{a, 2}-\frac{Q_{2}}{V_{2}} x_{a, 2} \\
& \dot{x}_{b, 2}=\mu_{b}\left(s_{2}\right) x_{b, 2}-\frac{Q_{2}}{V_{2}} x_{b, 2}
\end{aligned}
$$

with $s_{\text {out }}=\frac{Q_{1} s_{1}+Q_{2} s_{2}}{Q_{1}+Q_{2}}$.

The system of equations for the buffered chemostat is:

$$
\begin{aligned}
& \dot{s}_{1}=-\mu\left(s_{1}\right) x_{1}+\frac{Q_{1} S_{i n}+Q_{2} s_{2}-Q s_{1}}{V_{1}} \\
& \dot{x}_{1}=\mu\left(s_{1}\right) x_{1}+\frac{Q_{2} x_{2}-Q x_{1}}{V_{1}} \\
& \dot{s}_{2}=-\mu\left(s_{2}\right) x_{2}+\frac{Q_{2}}{V_{2}}\left(S_{i n}-s_{2}\right) \\
& \dot{x}_{2}=\mu\left(s_{2}\right) x_{2}-\frac{Q_{2}}{V_{2}} x_{2}
\end{aligned}
$$

with $s_{\text {out }}=s_{1}$. 